\title{
Fabrication of highly transparent and luminescent quantum dot/polymer nanocomposite for light emitting diode using amphiphilic polymer-modified quantum dots
}

\author{
Cheolsang Yoon ${ }^{\mathrm{a}, 1}$, Kab Pil Yang ${ }^{\mathrm{a}, 1}$, Jungwook Kim ${ }^{\mathrm{b}}$, Kyusoon Shin ${ }^{\mathrm{c}}$, Kangtaek Lee ${ }^{\mathrm{a}, *}$ \\ ${ }^{a}$ Department of Chemical and Biomolecular Engineering, Yonsei University, 50 Yonsei-ro, Seodaemun-gu, Seoul 03722, Republic of Korea ${ }^{\text {b }}$ \\ Department of Chemical and Biomolecular Engineering, Sogang University, 35 Baekbeom-ro, Mapo-gu, Seoul 04107, Republic of Korea ${ }^{c}$ \\ Advanced Institute of Research, Dongjin Semichem Co., Gyeonggido, Republic of Korea
}

\section{H I G H L I G H T S}

- $\mathrm{CdSe} @ \mathrm{ZnS} / \mathrm{ZnS}$ core/shell QDs were encapsulated by an amphiphilic polymer.

- QD/PDMS nanocomposite was fabricated by using encapsulated QDs as a crosslinker.

- Nanocomposite with uniform QD dispersion could be obtained even at high QD loading.

- Synthesized nanocomposite showed high transparency due to uniform QD dispersion.

- LED with the synthesized nanocomposite exhibited excellent luminous efficacy.

\section{ARTICLEINFO}

Keywords:

Quantum dots

Amphiphilic polymer

Surface modification

Dispersion

Light emitting diode

\begin{abstract}
A B S T R A C T
Herein we present the fabrication of a highly transparent and luminescent quantum dot (QD)/polymer nanocomposite for application in optoelectronic devices. First, we encapsulated CdSe@ZnS/ZnS core/shell QDs with an amphiphilic polymer, i.e., poly(styrene-co-maleic anhydride) (PSMA). By encapsulating QDs with PSMA instead of ligand exchange, the photoluminescence intensity of the QDs could be preserved even after surface modification. Next, the PSMA-modified QDs were used as crosslinkers for the aminopropyl-terminated polydimethylsiloxane (PDMS) resin in a ring-opening reaction between the maleic anhydride of the QDs and the diamines of the PDMS, producing polymer networks at a low curing temperature. This method afforded a nanocomposite with uniform dispersion of QDs even at high QD concentrations $(\sim 30 \mathrm{wt} \%)$ and superior optical properties compared to a nanocomposite prepared from unmodified QDs and commercial resin. Owing to these enhanced properties, the nanocomposite was used to fabricate a light emitting diode (LED) device, and the luminous efficacy was found to be highest at $1 \mathrm{wt} \%$.
\end{abstract}

\section{Introduction}

Fluorescent semiconductor nanocrystals, or quantum dots (QDs), have attracted great interest for the development of next-generation devices, such as light emitting diodes, solar cells, and luminescent solar concentrators, due to their excellent optical properties [1-9]. In particular, high color purity, photoluminescence quantum yield (PLQY), and photochemical stability of QDs make them suitable for alternative phosphors in light emitting diodes (LEDs) $[3,6]$. In white LED applications, for instance, QDs are used as color converters to convert blue light from an LED chip into green and red colors, thereby generating white light $[3,5]$. In these applications, QDs are usually in the form of a polymer nanocomposite consisting of QDs embedded in a polymer matrix, which protects them from harsh environments [10-13]. Among the various types of polymers used in LED applications, silicone-based polymers are frequently used because they exhibit excellent transmittance in visible light, thermal stability, and light-extraction efficiency [14-17]. When a siliconebased polymer is used as a matrix, however, it is difficult to fabricate highly transparent and luminescent nanocomposites due to the aggregation of QDs and

\footnotetext{
* Corresponding author.

E-mail address: ktlee@yonsei.ac.kr (K. Lee).

1 These authors contributed to this work equally.
} 
the high curing temperature $[18,19]$. Aggregation of QDs in a polymer matrix is usually accompanied by serious degradation of the optical properties because of light scattering by QD aggregates and luminescence quenching by Förster resonance energy transfer (FRET) between nearby QDs [20-22]. In addition, the high curing temperature of silicone polymer can damage the QD surface by detaching surface ligands and creating trap states, which reduce the quantum yield (QY) of individual QDs [14,23].

To improve the dispersion of QDs within the silicone matrix, various studies have been conducted [24-26]. For instance, surface ligands on QDs were replaced by ligands that were more compatible with the matrix. In our previous work, the dispersion of QDs in a poly(dimethylsiloxane) (PDMS) matrix was improved by modifying the surface of the QDs with the hydrophobic ligands containing a thiol anchoring group, but the increase in quantum efficiency (QE) of the nanocomposites was not significant [24]. Tao et al. fabricated a transparent QD/PDMS nanocomposite using bimodal PDMS-grafted QDs, but the concentration of QDs in the nanocomposite was low $(<1 \mathrm{wt} \%)$ and the QY of the PDMS-grafted QDs significantly decreased ( 50\%) after ligand exchange [25]. Both methods utilized the ligand exchange reaction that adversely affected surface passivation of the QDs, resulting in the reduction of QY. To circumvent this problem, encapsulation of QDs with amphiphilic polymer has been developed [26-28]. Instead of exchanging ligands on the QD surface, an amphiphilic polymer bearing both hydrophobic and hydrophilic groups was added to modify the surface. In this approach, the hydrophobic portion of the polymer intercalated with the surface ligands of the QD by hydrophobic interaction, thus retaining the original surface ligands and preserving their QY even after surface modification. But, most studies on modification of QDs with amphiphilic polymers have been focused on aqueous dispersion of QDs for biological applications $[27,28]$.

In this study, we report the fabrication of highly luminescent and transparent QD/PDMS nanocomposite films using QDs modified with an amphiphilic polymer. We used poly(styrene-co-maleic anhydride) (PSMA) to encapsulate QDs through hydrophobic interaction. The PSMA acted as a crosslinker for the matrix polymer in a ring-opening reaction between the maleic anhydride on the QDs and the amine end group of PDMS. This enhanced compatibility between the QDs and the PDMS matrix as well as improved the dispersion of the QDs. Using this method, we could fabricate a transparent QD-PSMA/PDMS nanocomposite film with uniform dispersion of QDs at high concentration (up to $30 \mathrm{wt} \%$ ) without a high-temperature curing step. Finally, the luminous efficacy of this method was compared with the conventional QD-PDMS nanocomposite in LED applications.

\section{Experimental}

\subsection{Materials}

Cadmium acetate $\left(\mathrm{Cd}(\mathrm{OAc})_{2}, 99.99 \%\right)$, cadmium oxide (CdO, 99.99\%), zinc acetate $\left(\mathrm{Zn}(\mathrm{OAc})_{2}, 99.99 \%\right)$ oleic acid (OA, 90\%), trioctylphosphine (TOP, 90\%), 1-octadecene (1-ODE), propylene glycol monomethyl ether acetate (PGMEA, 99.5\%), 1-dodecanethiol (1-DDT, 98\%), poly(styrene-co-maleic anhydride) cumene terminated (PSMA, $\mathrm{M}_{\mathrm{n}}$ : $\sim 1900$ by GPC), and bis(3aminopropyl) terminated poly(dimethylsiloxane) $\left(\mathrm{H}_{2} \mathrm{~N}-\mathrm{PDMS}-\mathrm{NH}_{2}, \mathrm{M}_{\mathrm{n}}\right.$ : 27,000) were purchased from Aldrich. Selenium (99.999\%, powder) and sulfur $(99.5 \%$, powder) were purchased from Alfa Aesar. Ethyl alcohol (99.99\%), acetone (99.99\%), n-hexane (95.0\%), and chloroform (99.99\%) were purchased from Duksan Chemical. All the chemicals were used without further purification.

\subsection{Synthesis of green- and red-emitting QDs}

Highly luminescent and stable green-emitting CdSe@ZnS/ZnS core/ shell QDs were synthesized by the previously reported method [29]. In a 50-mL three-neck flask, $0.14 \mathrm{mmol} \mathrm{Cd}(\mathrm{OAc})_{2}, 3.41 \mathrm{mmol} \mathrm{Zn}(\mathrm{OAc})_{2}$, and $7 \mathrm{~mL} \mathrm{OA}$ were added and degassed at $150{ }^{\circ} \mathrm{C}$ under vacuum until the vacuum level reached 100 mTorr. Temperature was then lowered to $100{ }^{\circ} \mathrm{C}$ and $15 \mathrm{~mL}$ of 1 ODE was injected into the flask, followed by degassing for $30 \mathrm{~min}$. After degassing, the temperature was elevated to $310{ }^{\circ} \mathrm{C}$ to obtain a transparent solution of $\mathrm{Cd}(\mathrm{OA})_{2}$ and $\mathrm{Zn}(\mathrm{OA})_{2}$, while maintaining Ar purging. Following this step, $2 \mathrm{~mL}$ TOPSeS, which was prepared by dissolving $5 \mathrm{mmol} \mathrm{Se}$ and 5 $\mathrm{mmol} \mathrm{S}$ in $5 \mathrm{~mL}$ TOP, was rapidly injected into the flask at $310{ }^{\circ} \mathrm{C}$ and allowed to react for $10 \mathrm{~min}$ for the growth of a CdSe@ZnS alloy core. For higher stability, the CdSe@ ZnS core was overcoated with a ZnS shell, by injecting $2.4 \mathrm{~mL} \mathrm{~S}$ source $(3.2 \mathrm{mmol} \mathrm{S}$ in $4.8 \mathrm{~mL} \mathrm{1-ODE})$ into the suspension of CdSe@ZnS cores. After $12 \mathrm{~min}$, the $5 \mathrm{~mL} \mathrm{Zn}$ source $\left(4.92 \mathrm{mmol} \mathrm{Zn}(\mathrm{OAc})_{2}\right.$ in $2 \mathrm{~mL} \mathrm{OA}$ and $8 \mathrm{~mL} \mathrm{ODE}$ ) was injected into the suspension. Then, $5 \mathrm{~mL} \mathrm{~S}$ precursor (19.3 mmol S in $10 \mathrm{~mL}$ TOP) was added into the reaction bath dropwise at a rate of $0.5 \mathrm{~mL} / \mathrm{min}$, and allowed to react for $20 \mathrm{~min}$. To complete the reaction, the reactor was quickly cooled to room temperature. The resulting QDs were precipitated by the addition of $4 \mathrm{~mL}$ hexane and $50 \mathrm{~mL}$ acetone, followed by centrifugation at $9000 \mathrm{rpm}$ for $10 \mathrm{~min}$ and redispersion in chloroform. The purification step was repeated three times, after which the QDs were dispersed in the chloroform for further use.

For the white LED experiment, red-emitting CdSe/CdZnS/ZnS QDs were synthesized using the previously reported method [30] with a modification. In a 250 -mL three-neck flask, $4 \mathrm{mmol} \mathrm{CdO}, 8 \mathrm{mmol} \mathrm{Zn}(\mathrm{OAc})_{2}$, and $20 \mathrm{~mL} \mathrm{OA}$ were mixed and degassed at $150{ }^{\circ} \mathrm{C}$ under vacuum (100 mTorr). After $1 \mathrm{~h}$, temperature was set to $50{ }^{\circ} \mathrm{C}$ and $100 \mathrm{~mL}$ 1-ODE was added to the mixture, followed by degassing at $100{ }^{\circ} \mathrm{C}$ for $1 \mathrm{~h}$. The mixture was then heated up to $300{ }^{\circ} \mathrm{C}$ with Ar purging, and $0.8 \mathrm{~mL}$ TOPSe, which was prepared by dissolving $1 \mathrm{mmol} \mathrm{Se}$ in $1 \mathrm{~mL}$ TOP, was rapidly injected to the mixture. After $1 \mathrm{~min}, 1.2$ $\mathrm{mL}$ 1-DDT was added dropwise at the rate of $1 \mathrm{~mL} / \mathrm{min}$. After $20 \mathrm{~min}, 4 \mathrm{~mL}$ TOP dissolving $4.67 \mathrm{mmol} \mathrm{S}$ was injected to passivate CdSe/CdZnS QDs with $\mathrm{ZnS}$ shell for $10 \mathrm{~min}$. The temperature of reactor was lowered to room temperature, and QDs were precipitated with hexane/acetone, followed by centrifugation and redispersion in chloroform. The as-synthesized red-emitting QDs were dispersed in chloroform for white LED experiments.

2.3. Surface modification by amphiphilic polymer and preparation of QDPSMA/PDMS nanocomposite

Surface modification of the QDs with amphiphilic polymer (PSMA) was conducted using a hydrophobic interaction between the surface ligands of the QDs and the PSMA. Different amounts of PSMA were added to a 2-mL QD suspension in chloroform. The QD/polymer suspension was stirred for $6 \mathrm{~h}$, leading to a dissolution of PSMA and formation of PSMA-modified QDs (QDPSMA).

To fabricate the QD-PSMA/PDMS nanocomposite, amine terminatedPDMS $\left(\mathrm{H}_{2} \mathrm{~N}-\mathrm{PDMS}-\mathrm{NH}_{2}\right)$ was mixed with the QD-PSMA suspension at room temperature, enabling a crosslinking reaction between PDMS and the amphiphilic polymer on the QDs. For comparison, nanocomposite was also prepared by using commercially available PDMS polymer (Sylgard-184, Dow Corning), where the QDs or QD-PSMA were mixed with $1 \mathrm{~g}$ base polymer and $0.1 \mathrm{~g}$ curing agent. The mixture was vacuum-dried for $1 \mathrm{~h}$ at room temperature to remove the solvent and air bubbles, and cured in a convection oven at $150{ }^{\circ} \mathrm{C}$ for $2 \mathrm{~h}$, resulting in the QD/Sylgard or QD-PSMA/Sylgard nanocomposite.

\subsection{LED applications}

For LED experiments, the green-emitting QD-PSMA/PDMS mixture was placed on a blue LED chip (Dae-Kwang Illumination, $\lambda_{\mathrm{ex}}=450 \mathrm{~nm}$ ) surface mount device (SMD), followed by curing at $150{ }^{\circ} \mathrm{C}$. For comparison, LEDs were prepared using the QD/Sylgard nanocomposite. White LED device was also fabricated using the QDPSMA/PDMS containing CdSe@ZnS/ZnS green QDs and CdSe/CdZnS/ ZnS red QDs at the weight ratio of 10:1. The luminous properties of the LEDs were then measured by the integrating sphere equipped with the LED test apparatus. 
For a thermal stability test, luminous efficacy of the fabricated QDPSMA/PDMS LED device was measured in a convection oven at $100{ }^{\circ} \mathrm{C}$ for 15 days.

\subsection{Characterization}

The size and morphology of the QDs were determined using transmission electron microscopy (TEM, JEM-2100, JEOL) and quasi-elastic light scattering (QELS, Nano-ZS, Malvern). The UV-Vis absorption spectra of the QDs and transmittance of the nanocomposite films were obtained using a spectrophotometer (V-730, JASCO). The emission spectra were obtained using a Perkin-Elmer LS-55 photoluminescence (PL) spectrometer. The dispersion states of QD-PSMA/PDMS and QD/ Sylgard nanocomposites were observed with a confocal microscope (LSM-880, ZEISS). The quantum yield was measured with a spectrofluorometer (FP-8500, JASCO) and an integrating sphere (ILF-835, JASCO). The luminous and color conversion efficiencies of the LEDs were estimated using an integrating sphere with a 450-nm Xe laser as an excitation energy source (QE-1000, Otsuka Electronics).

\section{Results and discussion}

\subsection{Characterization of the as-synthesized and surface-modified QDs}

Highly luminescent green-emitting CdSe@ZnS/ZnS QDs capped with OA and TOP were synthesized by the previously reported method [29], and their surfaces were modified with the PSMA to produce QDPSMA. PL spectra in Fig. 1(a) show that the PL intensity and $\lambda_{\max }$ of the QD-PSMA remained virtually constant even after surface modification. It has been reported that PL intensity of QDs usually decreases after ligand exchange due to detachment of the original surface ligands. Coating amphiphilic polymers on the QD surface, however, retained the original ligands on the surface and hence preserved the PL intensity and $\lambda_{\max }$. After surface modification with PSMA, the average hydrodynamic diameter of QDs from the QELS, slightly increased from 18.5 \pm $2.7 \mathrm{~nm}$ to $23.26 \pm 4.2 \mathrm{~nm}$, while the TEM did not show a noticeable size change (Fig. 1(b)). The discrepancy between the QELS and TEM sizes suggests that the increase in the hydrodynamic diameter can be attributed to the encapsulation by PSMA. Moreover, when a polar solvent such as PGMEA was added to the as-synthesized QD suspension, aggregation of QDs occurred because of the hydrophobic nature of the QD surfaces. However, PSMA-capped QDs remained colloidally stable in the PGMEA solution, supporting successful modification with the PSMA as shown in Fig. 1(c).

\subsection{Preparation of the QD-PSMA/PDMS nanocomposites}

QD-PSMA/PDMS nanocomposite was prepared by mixing QD-PSMA and amine terminated-PDMS polymer. The mixture gelled within $2 \mathrm{~h}$, which was caused by the crosslinking reaction between the MA group on the QD surface and the amine group of the PDMS. Note that the QDPSMA acted as a crosslinker for the PDMS polymer (Fig. 2).

To understand the effect of the PSMA amount on the crosslinking reaction, molar ratios of the MA on the QD-PSMA to the $\mathrm{NH}_{2}$ of the PDMS were varied from 0.1 to 14.8 while the QD concentration was

(a)

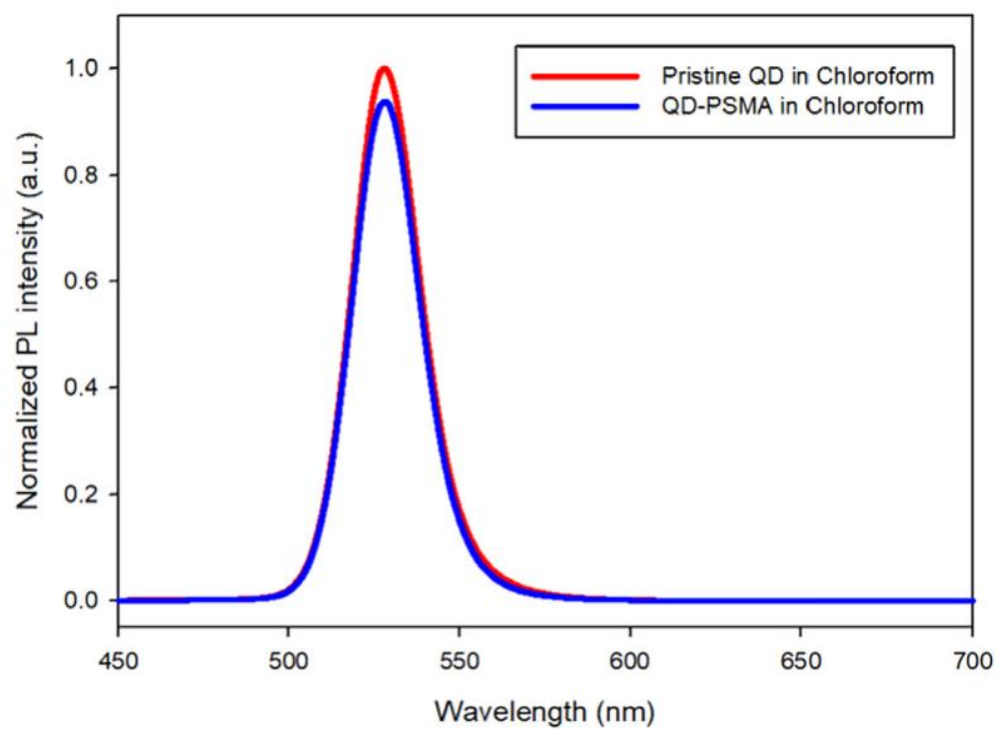

(b)

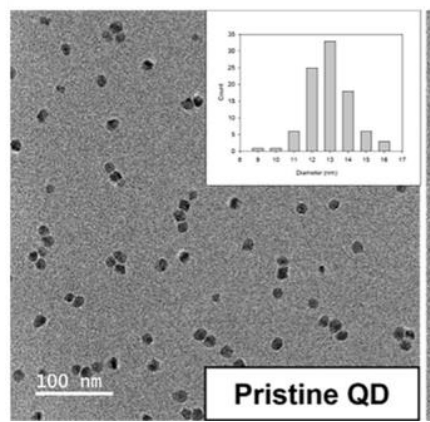

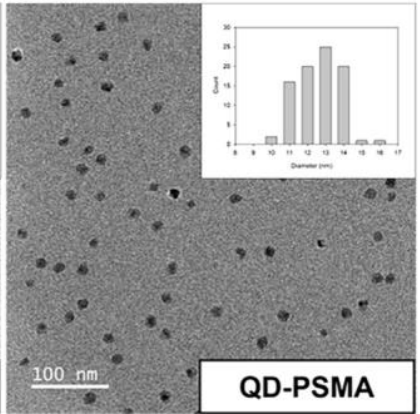

(c)

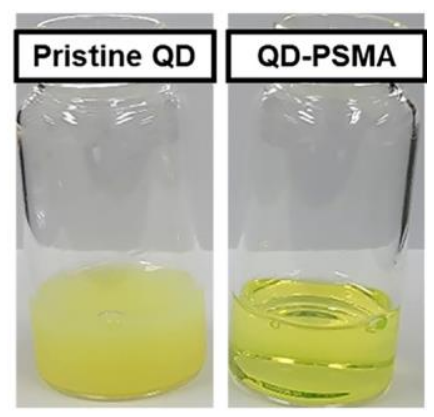

Fig. 1. Properties of the as-synthesized QDs and QD-PSMA: (a) PL intensity, (b) TEM images, and (c) colloidal stability in PGMEA. 


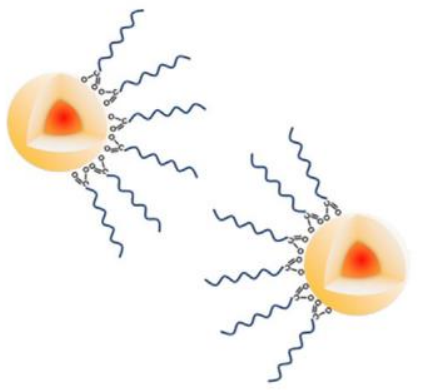

QD with hydrocarbon chain ligand<smiles>CCC(CC)C(C)(C)C1CCCC1</smiles>

Surface modification

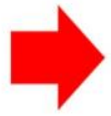

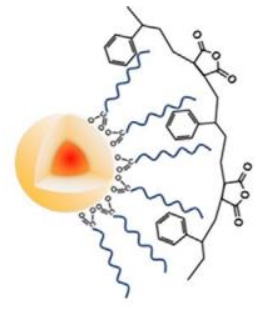

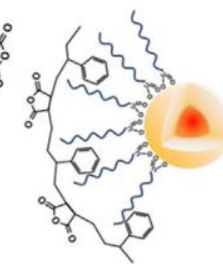

Hydrophobic attraction between QD and amphiphilic polymer

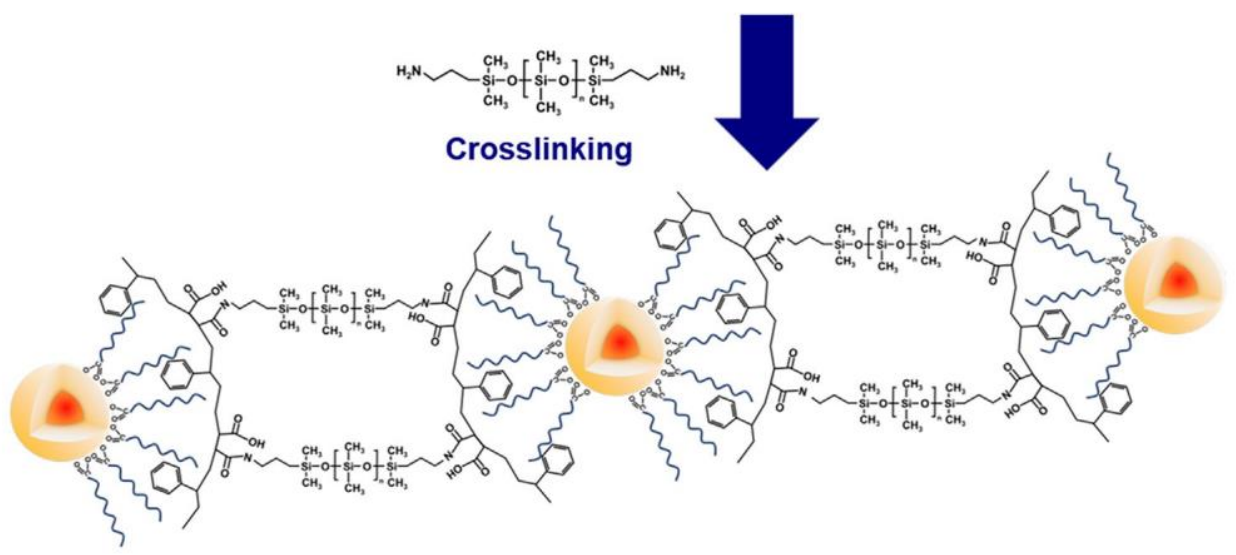

Fabricated QD-PSMA/PDMS nanocomposite

Fig. 2. Schematic representation of crosslinking mechanism between the surface-modified QDs and the amine-terminated PDMS.

kept constant at $1 \mathrm{wt} \%$. Fig. S1 shows that gelation did not occur when the $\mathrm{MA} / \mathrm{NH}_{2}$ ratio was lower than 0.4 or greater than 3.7, indicating an optimal amount of PSMA for gelation. If the MA/ $\mathrm{NH}_{2}$ ratio was either too low or too high, the extent of the crosslinking reaction in the PDMS network was not high enough for gelation, resulting in a fluidic mixture. From the above results, the molar ratio of $\mathrm{MA} / \mathrm{NH}_{2}$ was set to 2.2, and QD-PSMA/PDMS nanocomposite films were fabricated by coating a mixture of QD-PSMA and PDMS on a glass substrate, followed by solvent evaporation. For comparison, nanocomposite film was also prepared using the unmodified QDs and commercial polymer (QD/Sylgard).

\subsection{Optical properties of QD-PSMA/PDMS nanocomposites}

We compared optical properties of the QD-PSMA/PDMS and the QD/Sylgard nanocomposite films. Fig. 3 shows that transparent QDPSMA/PDMS films could be obtained with a wide range of QD concentrations (0.1-30 wt\%). However, the QD/Sylgard film was translucent even at the QD concentration of $1 \mathrm{wt} \%$. Generally, a reduction in transparency of the nanoparticle-based nanocomposite is attributed to the aggregation phenomenon, which is known to cause light scattering and a redshift of $\lambda_{\max }$ in the emission spectrum. The QD-PSMA/PDMS films remained transparent even when the QD concentration was very high (30 wt\%), and only a slight redshift was observed as the QD concentration increased (Fig. 4(a)). Alternatively, the QD/Sylgard film was translucent, due to light scattering, and the redshift was evident even at $1 \mathrm{wt} \%$. These results suggest that QDs were distributed in the form of aggregates in the QD/Sylgard film.

We also compared transmittances of QD-PDMS nanocomposite films by UV-Vis spectroscopy. In particle-embedded nanocomposites, several factors can affect transmittance. According to the Rayleigh scattering theory, the loss of transparency of nanocomposites results from light scattering by spherical nanoparticles with radius $r$ and volume fraction $\varphi_{p}$ as follows:

$$
T=\exp \left[\frac{\left.\left.\lambda_{4} \quad\left\{\begin{array}{l}
)_{2}-1 \\
\left(n n_{p} m /\right)_{2}+2
\end{array}\right\}\right) \|\right]}{32 \pi \phi x r n 4 p 3 m 4\left(n n_{p m} /\right.}\right.
$$

where $\mathrm{T}$ is the transmittance of the nanocomposites, $\lambda$ is the wavelength of light, $\mathrm{x}$ is the optical path length, and $\mathrm{n}_{\mathrm{p}}$ and $\mathrm{n}_{\mathrm{m}}$ are the refractive indices of the nanoparticle and polymer matrix, respectively. In this work, all the parameters were identical except the size of particles in the films. Generally, the particle size should be smaller than approximately one-tenth of the wavelength of light to minimize the loss of transparency by light scattering. Thus, aggregation of QDs can lead to significant light scattering and hence reduce transparency of the nanocomposite film. Fig. 4(b) shows that the QD-PSMA/PDMS films exhibited much higher transmittance than the QD/Sylgard film at all wavelengths regardless of the QD concentration, because aggregation caused significant light scattering and reduction in transmittance in the QD/Sylgard film. In addition, increasing QD concentration decreased the transmittance in the QD-PSMA/PDMS films. Because the light scattering was negligible in the QD-PSMA/PDMS film (Fig. 3), transmittance was mainly governed by absorption by the QDs, decreasing transmittance with the QD concentration.

Using an integrating sphere, we measured QY of QDs after fabrication of QD-PSMA/PDMS nanocomposite, and found that QY decreased from $81 \%$ to $55 \%$. There have been several studies that reported a QY drop after incorporation of QDs within polymer composites [31-33]. We suspect that QDs were oxidized during dispersion and curing processes, resulting in luminescence quenching. 


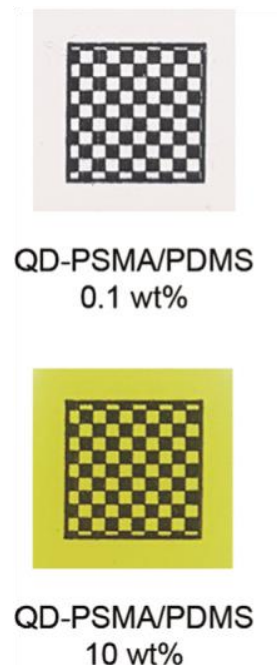

$10 w t \%$
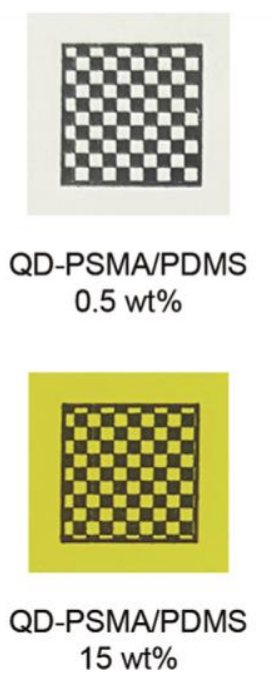

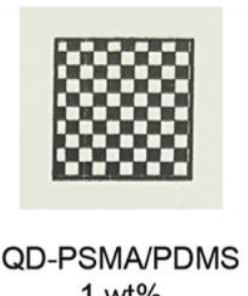

$1 \mathrm{wt} \%$

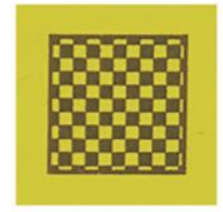

QD-PSMA/PDMS
30 wt\%

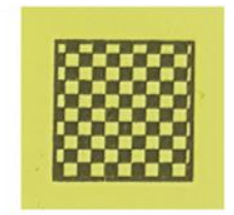

\section{QD-PSMA/PDMS}

$5 \mathrm{wt} \%$

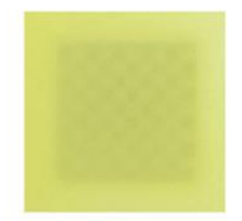

QD-Sylgard 1 wt\%

Fig. 3. Pictures of QD-PSMA/PDMS nanocomposites with different QD concentrations when MA/NH2 ratio was 2.2 and QD/Sylgard nanocomposite (QD 1 wt\%) on glass substrates.

\subsection{Dispersion states of QDs within nanocomposite films}

To confirm the relation between the optical properties and dispersion state of the nanocomposite films, we directly observed dispersion states of QDs in QD-PSMA/PDMS and QD/Sylgard films with the confocal microscope and TEM. Fig. 5(a) and (b) show that the distribution of QDs was uniform in the QD-PSMA/PDMS, while in the QD/Sylgard there were mostly micro-sized fluorescent particles and voids (no fluorescence) due to significant aggregation. We also prepared focused ion beam (FIB) samples of QD-PSMA/PDMS for TEM analysis, which showed that QDs existed as isolated nanoparticles without aggregation even at high concentration (Fig. 5(c) and (d)).

The discrepancy in the dispersion states of QDs can be explained as follows. In the QD/Sylgard nanocomposite, van der Waals attraction between QDs was dominant, causing serious aggregation of QDs in the polymer matrix. In the QD-PSMA/PDMS nanocomposite, however, van der Waals attraction between QDs was reduced because the QDs were isolated by the PSMA polymer encapsulation layer, which also provided steric stabilization. Furthermore, the crosslinking reaction of the QDPSMA with the amine-terminated PDMS caused QDs to be enclosed by the PDMS network. Note that the amine-terminated PDMS in this study had a molecular weight of 27,000 , which was sufficiently high to act as a spacer to prevent the aggregation of QDs. To determine whether the PSMA encapsulation layer or the crosslinking reaction was more important in uniform dispersion of QDs, the PSMA-modified QDs were dispersed in the commercial PDMS polymer without amine groups (QDPSMA/Sylgard). Fig.

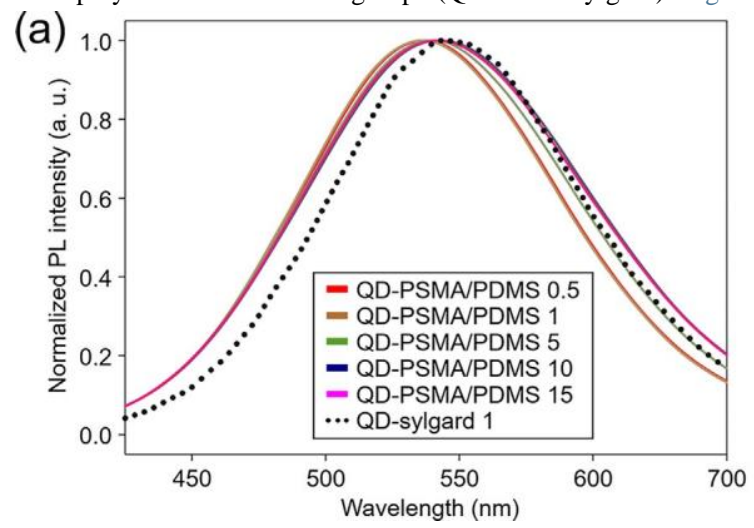

$\mathrm{S} 2$ shows that without amine groups, QDs exhibited significant aggregation even at $1 \mathrm{wt} \%$, confirming that the crosslinking reaction played a dominant role in improving dispersion of QDs in the QD-PSMA/PDMS nanocomposite.

\subsection{LED application of QD/PDMS nanocomposites}

LED chips were prepared by curing QD-PSMA/PDMS at various QD concentrations $(0.1,0.5,1,5,10,15 \mathrm{wt} \%)$ and 1 wt\% QD/Sylgard nanocomposite on the blue LED SMD chips. Note that it was possible to fabricate an LED chip with the QD-PSMA/PDMS even at high concentration while poor dispersion of QDs in the QD/Sylgard prevented the fabrication of the LED chip at high concentration. We compared luminous efficacies of the LED chips using an integrating sphere (with the LED equipped). When the LEDs were operated at $60 \mathrm{~mA}$, the luminous efficacy of the QD-PSMA/PDMS was higher than that of the $1 \mathrm{wt} \% \mathrm{QD} /$ Sylgard nanocomposite even at much higher QD concentration (15 wt \%, Fig. 6(a)). The luminous efficacy of QDPSMA/ PDMS nanocomposite was highest at $1 \mathrm{wt} \%$. Above $1 \mathrm{wt} \%$, reabsorption of emitted light by QDs and FRET among QDs became significant due to a decrease in the interparticle distance, which decreased the luminous efficacy. In addition, electroluminescence spectra of the LED chips using the QD-PSMA/PDMS and QD/Sylgard nanocomposites at the same QD concentration (1 wt\%) were obtained. Fig. 6(b) shows that an LED with the QD-PSMA/PDMS exhibited higher emission intensity than that with the QD/Sylgard even though much more blue light remained, which suggests that the QD-PSMA/PDMS nanocomposite could convert blue light to emitted light more efficiently, leading to higher luminous

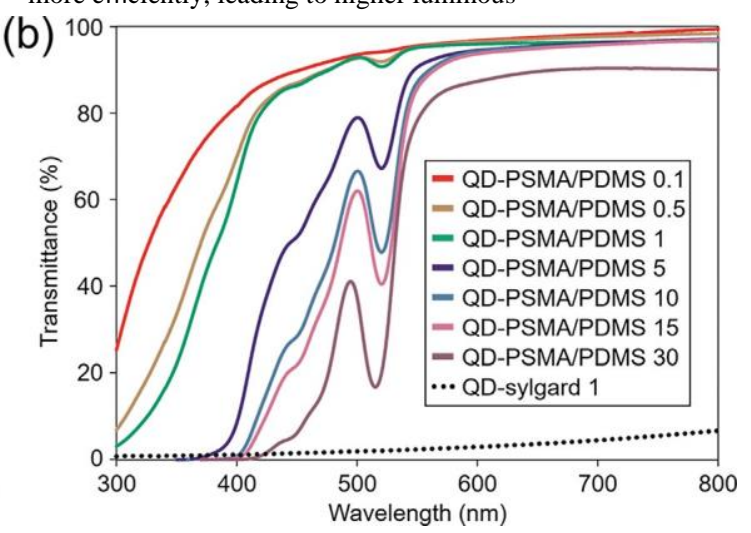

Fig. 4. Photoluminescence spectra (a) and transmittance (b) of nanocomposite films (numbers indicate wt\%). 
(a)

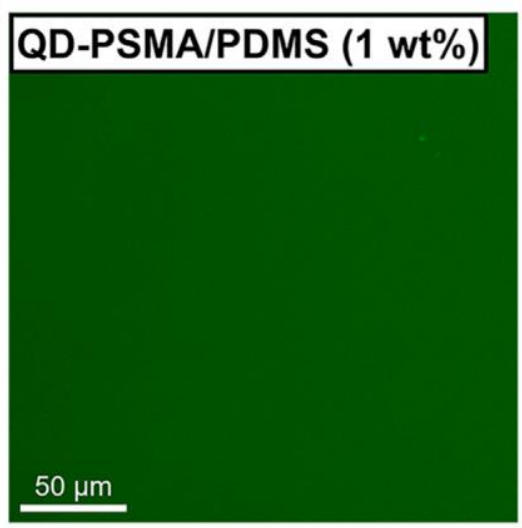

(c)

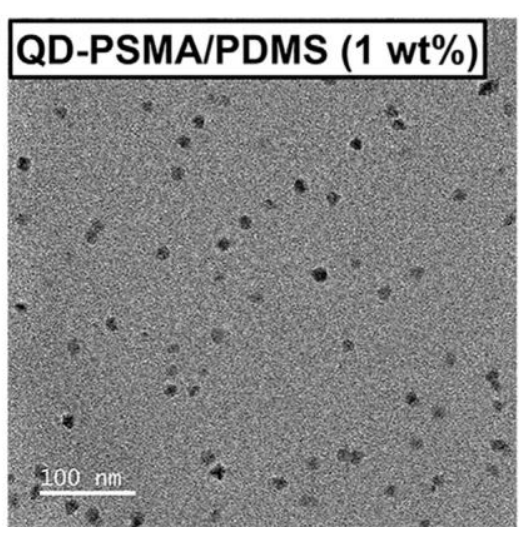

(b)

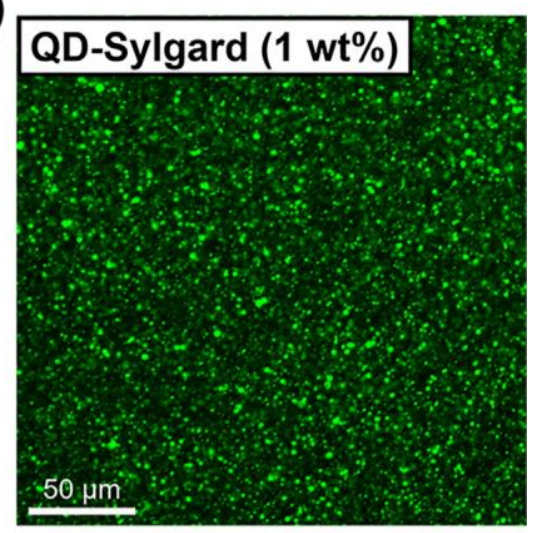

(d)

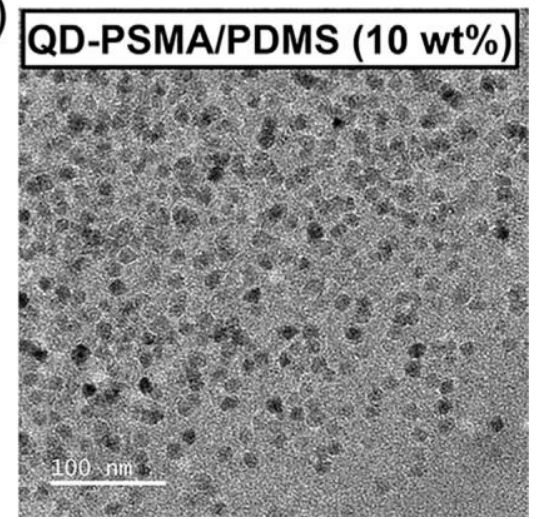

Fig. 5. Confocal microscope images of QDs within QD-PSMA/PDMS (a) and QD/Sylgard nanocomposites (b), and TEM images of QD-PSMA/PDMS with different QD

concentrations ((c), (d)).

efficacy. Conversely, an LED with QD/Sylgard exhibited not only lower emission intensity but also a significant redshift in $\lambda_{\max }(\sim 8 \mathrm{~nm})$. This result can be explained by aggregation of QDs in the QD/Sylgard nanocomposite that blocked light propagation and generated backscattering and reabsorption, causing a reduction in luminous efficacy and a redshift in $\lambda_{\max }$. The calculated color conversion efficiency of the LED with the QD-PSMA/PDMS was also much higher (17.1\%) than that with the QD/Sylgard (5.1\%). We also tested a thermal stability of the LED with the QD-PSMA/PDMS, and confirmed that luminous efficacy could be preserved (> 94\%) even after an exposure to high temperature $\left(100^{\circ} \mathrm{C}\right)$ for 15 days (Fig. 7 (a)). We believe that effective isolation of the PSMA-encapsulated QDs within PDMS resin using the crosslinking reaction could prevent QDs from oxidation at high temperature. Finally, we fabricated a white LED using the QD-PSMA/PDMS with both greenand red-

(a)

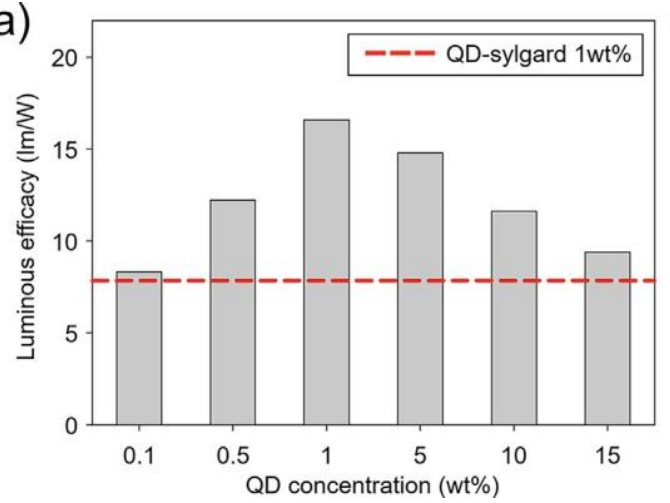

emitting QDs. Fig. 7(b) shows that white color could be obtained (color coordinate: $\mathrm{x}=0.363, \mathrm{y}=0.3564$ ) using this method, suggesting its potential for white LED applications.

\section{Conclusions}

To utilize QDs as a new-generation material in optoelectronic devices, meet requirements for various device morphologies, and keep QDs from direct exposure to extreme conditions such as high humidity or temperature, it is necessary to fabricate QD-polymer nanocomposites from colloidal QDs. In this work, we presented a novel method to fabricate a QD-polymer nanocomposite that possesses superior optical properties to those with conventional resins. In particular, QDs were encapsulated with the amphiphilic polymer (PSMA), which then acted

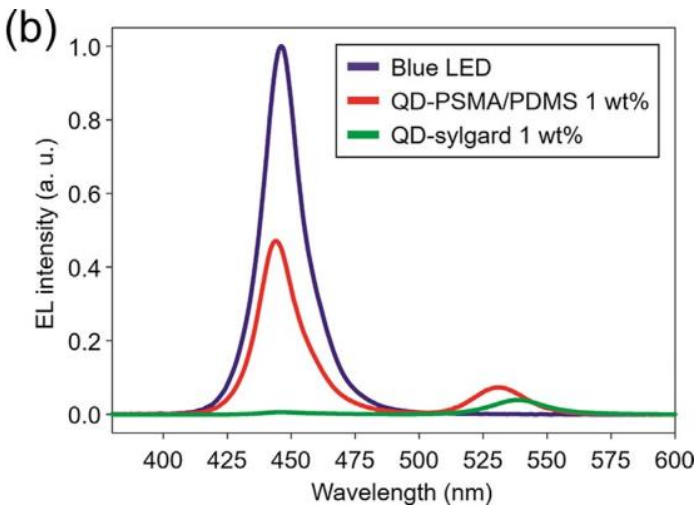

Fig. 6. Luminous efficacy of the LED chips using QD-PSMA/PDMS with different QD concentrations and QD/Sylgard of 1 wt $\%$ of QD at a forward bias current of $60 \mathrm{~mA}$ (a), and electroluminescence (EL) spectra of the LED chips using QD-PSMA/PDMS $1 \mathrm{wt} \%$ and QD/Sylgard at $1 \mathrm{wt} \%$ (b). 
(a)

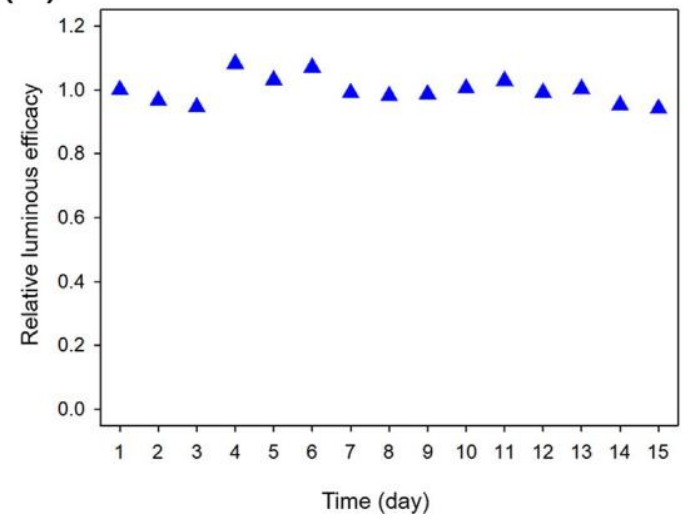

(b)

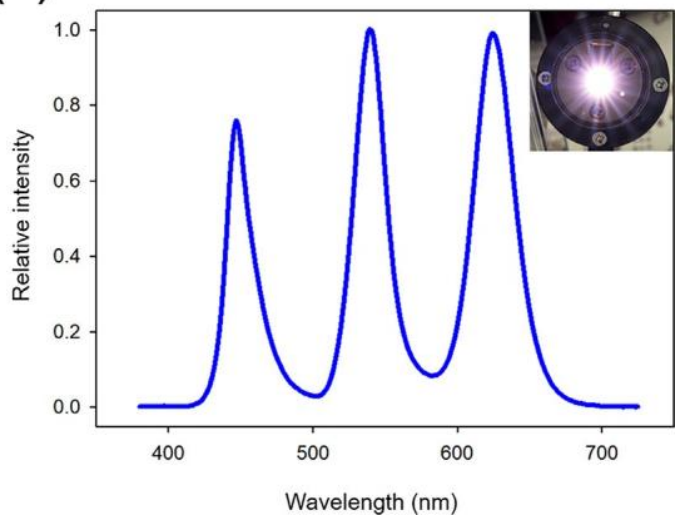

Fig. 7. Changes in relative luminous efficacy of LED with QD-PSMA/PDMS nanocomposite at $100{ }^{\circ} \mathrm{C}$ (a), and electroluminescence spectra of the fabricated white LED with QD-PSMA/PDMS nanocomposite (b).

as a crosslinker for the amine-terminated PDMS polymer to produce a nanocomposite with a uniform dispersion of QDs. The resulting nanocomposite exhibited higher transparency and luminous efficacy than that with unmodified QDs and commercial resin. These results can be used to design a new device that requires high transparency and efficiency at high QD concentration.

Acknowledgements

This work was supported by National Research Foundation of Korea (NRF) (grant nos. NRF-2018R1A5A1024127 and 2017R1A2B4007534) and the Ministry of Trade, Industry \& Energy (grant no. 10079347) through the Development of Materials and Core-technology for Future Display support program.

\section{Appendix A. Supplementary data}

Supplementary data to this article can be found online at https:// doi.org/10.1016/j.cej.2019.122792.

\section{References}

1. Zhang, Y. F.; Xu, Z.; Zhang, F. J.; Wang, Y.; Zhao, S. L., White organic light emitting device with dyestuff DCJTB mended in polymer. Spectroscopy and Spectral Analysis 2008, 28 (4), 760-762.

2. Watanabe, K.; Sakamoto, T.; Taguchi, M.; Fujiki, M.; Nakano, T., A chiral pi-stacked vinyl polymer emitting white circularly polarized light. Chem. Commun. 2011, 47 (39), 10996-10998.

3. Wang, R.; Peng, J.; Qiu, F.; Yang, Y. L., Enhanced white-light emission from multiple fluorophores encapsulated in a single layer of diblock copolymer micelles. Chem. Commun. 2011, 47, 2787-2789

4. Prakash, A.; Katiyar, M., White polymer light emitting diode using blend of fluorescent polymers. 16th International Workshop on Physics of Semiconductor Devices 2012, 8549.

5. Park, J. J.; Park, T. J.; Jeon, W. S.; Kim, S. Y.; Lee, Y. K.; Jang, J.; Kwon, J. H., White Polymeric Light-Emitting Diodes Based on Doping of an Orange Ir Complex in a Fluorene Blue Polymer Host. Mol. Cryst. Liq. Cryst. 2009, 498, 290-297.

6. Park, J. J.; Park, T. J.; Jeon, W. S.; Kim, S. Y.; Lee, Y. K.; Jang, J.; Kwon, J. H., White polymeric light-emitting diodes based on doping of an orange Ir complex in a fluorene blue polymer. Idw' 07 : Proceedings of the 14th International Display Workshops, Vols 1-3 2007, 1033-1036.

7. Nicolai, H. T.; Hof, A. J.; Blom, P. W. M., Charge transport in white light-emitting polymers. Organic Optoelectronics and Photonics Ii 2006, 6192.

8. Nicolai, H. T.; Hof, A.; Blom, P. W. M., Device Physics of White Polymer Light-Emitting Diodes. Adv. Funct. Mater. 2012, 22 (10), 20402047.

9. Li, B. N.; Liu, L.; Fu, G. R.; Zhang, Z.; Li, H. Y.; Lu, X.; Wong, W. K.; Jones, R. A., Color-tunable to direct white-light and application for white polymer light emitting diode (WPLED) of organo-Eu3+- and organo-Tb3+doping polymer. J. Lumin. 2017, 192, 1089-1095.

10. Shen, F. Z.; He, F.; Lu, D.; Xie, Z. Q.; Xie, W. J.; Ma, Y. G.; Hu, B., Bright and colour stable white polymer light-emitting diodes. Semiconductor Science and Technology 2006, 21 (2), L16-L19.

11. Kim, C.; Gwon, Y. J.; Kim, J.; Lee, T. S., Synthesis of fluorescent conjugated polymer nanoparticles and their immobilization on a substrate for white light emission. Polym Chem-Uk 2018, 9 (48), 5671-5679.

12. Amin, G.; Zaman, S.; Zainelabdin, A.; Nur, O.; Willander, M., ZnO nanorods-polymer hybrid white light emitting diode grown on a disposable paper substrate. Physica Status Solidi-Rapid Research Letters 2011, 5 (2), 7173.

13. Taudt, C.; Baselt, T.; Oreski, G.; Hirschl, C.; Koch, E.; Hartmann, P., Cross-linking characterization of polymers based on their optical dispersion utilizing a white-light interferometer. Optical Measurement Systems for Industrial Inspection Ix 2015, 9525.

14. Song, H. J.; Shin, G. J.; Choi, K. H.; Lee, S.; Moon, D. K., White polymer light emitting diode materials introducing dendritic quinoxaline derivative: Synthesis, optical and electroluminescent properties. Synthetic Metals 2014, 190, 1-7.

15. Lee, H. K.; Kim, T. H.; Park, J. H.; Kim, J. K.; Park, O. O., Whitelight-emitting diodes using miscible polymer blend doped with phosphorescent dye. Organic Electronics 2011, 12 (6), 891-896. 16. Kim, J. H.; Song, W. S.; Yang, H., Color-converting bilayered composite plate of quantum-dot-polymer for high-color rendering white light-emitting diode. Opt. Lett. 2013, 38 (15), 2885-2888.

17. Kassamakov, I.; Ojala, K.; Salmia, A.; Haeggstrom, E.; Aaltonen, J.; Huber, A.; Saarikko, H.; Osterberg, M.; Oinonen, M., Characterization of dents and grooves on polymer films using scanning white light interferometry. Optical Micro- and Nanometrology in Microsystems Technology 2006, 6188 .

18. Inoue, A.; Hosokawa, T.; Haishi, M.; Ohtani, N., 4-(dicyanomethylene)2-methyl-6-(p-dimethylaminostyryl)-4H-pyran (DCM)-doping density dependence of luminescence spectra and white emission in polymer lightemitting diodes. Physica Status Solidi C - Current Topics in Solid State Physics, Vol 6, No 1 2009, 6 (1), 334-337.

19. Hu, B.; Yao, C.; Huang, X. R., Designing of the White-Light Emission from a Single-Polymer System: Quantum Theoretical Study. Polymer Science Series A 2011, 53 (11), 1097-1105.

20. Fan, L. M.; Fan, W. L.; Li, B.; Zhao, X.; Zhang, X. T., W-shaped 1,3di(2,4-dicarboxyphenyl)benzene based lanthanide coordination polymers with tunable white light emission. New J. Chem. 2016, 40 (12), 10440-10446. 21. da Silva, M. A. T.; Thomazini, E. F.; Albertini, M.; Renzi, W.; Franchello, F.; Dias, I. F. L.; Duarte, J. L.; Pocas, L. C.; Lourenco, S. A., Characterization of digital textile printing and polymer blend (PFO-

DMP:P3HT) for application in manufacture of organic diodes emitting white light - WOLEDS. Optical Materials 2016, 62, 119-131.

22. Chen, M. X.; Sun, R. Y.; Ye, Y. C.; Tang, H. J.; Dong, X. Y.; Yan, J. L.; Wang, K. M.; Zhou, Q.; Wang, Z. L., Application of a novel red-emitting cationic iridium(III) coordination polymer in warm white light-emitting diodes. Optical Materials 2018, 76, 141-146.

23. Wang, R.; Peng, J.; Qiu, F.; Yang, Y. L.; Xie, Z. Y., Simultaneous blue, green, and red emission from diblock copolymer micellar films: a new approach to white-light emission. Chem. Commun. 2009, (44), 6723-6725. 
24. Hrma, M.; Sichova, K.; Svoboda, J.; Vohlidal, J., Assembling of bis(tpy)fluorenes with $\mathrm{Zn} 2+$ and $\mathrm{Fe} 2+$ ions into metallo-supramolecular polymers with highly efficient white-light emission. Polymer 2017, 122, 2233.

25. Chitara, B.; Bhat, S. V.; Vivekchand, S. R. C.; Gomathi, A.; Rao, C. N. R., White-light sources based on composites of GaN nanocrystals with conducting polymers and nanophosphors. Solid State Communications $\mathbf{2 0 0 8}$, 147 (9-10), 409-413.

26. Cheng, G.; Fei, T.; Zhao, Y.; Ma, Y. G.; Liu, S. Y., White phosphorescent polymer light-emitting devices based on a wide band-gap polymer derived from 3,6-carbazole and tetraphenylsilane. Organic Electronics 2010, 11 (3), 498-502.

27. Chen, S. A.; Chang, E. C.; Chuang, K. R.; Chao, C. I.; Wei, P. K.; Fann, W. S., Conjugated polymer blends as emitting layer for white light led. Abstr. Pap. Am. Chem. Soc. 1998, 215, U392-U392.

28. Zhen, H. Y.; Xu, W.; King, W.; Chen, Q. L.; Xu, Y. H.; Jiang, J. X.; Peng, J. B.; Cao, Y., White-light emission from a single polymer with singlet and triplet chromophores on the backbone. Macromol. Rapid Commun. 2006, 27 (24), 2095-2100

29. Zhang, T. H.; Gong, Z. C.; Que, L., A white-light source operated polymer-based micromachined Fabry-Perot chemo/biosensor. 2009 4th Ieee International Conference on Nano/Micro Engineered and Molecular Systems, Vols 1 and 2 2009, 181-184.

30. Willander, M.; Nur, O.; Zaman, S.; Zainelabdin, A.; Bano, N.; Hussain, I., Zinc oxide nanorods/polymer hybrid heterojunctions for white light emitting diodes. J. Phys. D: Appl. Phys. 2011, 44 (22).

31. Wang, Z. W.; Gao, D. Z.; Ma, X. J.; Meng, J., White-Light Interferometry for Measuring Fuel Pressure in Icf Polymer-Microsphere Targets. Fusion Sci. Technol. 2014, 66 (3), 432-437.

32. Wang, B. Z.; Zhang, X. P.; Liu, H. M., White-light-emitting diode based on a single-layer polymer. Aip Advances 2013, 3 (5).

33. Tian, L. L.; Zhang, W.; Yang, B.; Lu, P.; Zhang, M.; Lu, D.; Ma, Y. G.; Shen, J. C., Zinc(II)-induced color-tunable fluorescence emission in the pi-conjugated polymers composed of the bipyridine unit: A way to get whitelight emission. J. Phys. Chem. B 2005, 109 (15), 6944-6947.

34. Tang, K. C.; Tseng, S. R.; Li, W. S.; Meng, H. F.; Horng, S. F.; Hsu, C. S., Broad band and white phosphorescent polymer light-emitting diodes in multilayer structure. Synthetic Metals 2008, 158 (7), 287-291.

35. Sun, C.; Zhang, Y.; Sun, K.; Reckmeier, C.; Zhang, T. Q.; Zhang, X. Y.; Zhao, J.; Wu, C. F.; Yu, W. W.; Rogach, A. L., Combination of carbon dot and polymer dot phosphors for white light-emitting diodes. Nanoscale 2015, 7 (28), 12045-12050.

36. Roberts, R. J.; Le, D.; Leznoff, D. B., Color-Tunable and White-Light Luminescence in Lanthanide Dicyanoaurate Coordination Polymers. Inorg. Chem. 2017, 56 (14), 7948-7959.

37. Qin, L. J.; Zhu, Y. C.; Yang, H.; Ding, L.; Sun, F.; Shi, M.; Yang, S. P., White-light phosphorescence from binary coordination polymer nanoparticles. Mater. Chem. Phys. 2013, 139 (2-3), 345-349.

38. Ovens, J. S.; Christensen, P. R.; Leznoff, D. B., Designing Tunable White-Light Emission from an Aurophilic Cu-I/Au-I Coordination Polymer with Thioether Ligands. Chemistry-a European Journal 2016, 22 (24), 82348239 .

39. Niu, Y. H.; Liu, M. S.; Ka, J. W.; Bardeker, J.; Zin, M. T.; Schofield, R.; Chi, Y.; Jen, A. K. Y., Crosslinkable hole-transport layer on conducting polymer for high-efficiency white polymer light-emitting diodes. Adv. Mater. 2007, 19 (2), 300-+.

40. Niu, W. Y.; Sun, J. W.; Yan, P. F.; Li, Y. X.; An, G. H.; Li, G. M., 2D 1-Di-toluoyl-tartaric acid Lanthanide Coordination Polymers: Toward Singlecomponent White-Light and NIR Luminescent Materials. Chemistry-an Asian Journal 2016, 11 (4), 555-560.

41. Nam, G. H.; Park, I. K., CdSe Quantum dot-conducting polymer hybrid structure for Phosphor-free white light-emitting diodes. Journal of the Korean Physical Society 2015, 66 (5), 785-789.

42. Meng, L. C.; Lou, Z. D.; Yang, S. Y.; Hou, Y. B.; Teng, F.; Liu, X. J.; $\mathrm{Li}$, Y. B., White organic light-emitting diodes based on a combined electromer and monomer emission in doubly-doped polymers. Chinese Physics B 2012, 21 (8). 